\title{
Immune-related matrisomes are potential biomarkers to predict the prognosis and immune microenvironment of glioma patients through bioinformatics analysis and experimental verification
}

Xiaobing Jiang ( $\square$ m201975766@hust.edu.cn )

Huazhong University of Science and Technology

Hao Yu

Huazhong University of Science and Technology

Minjie Wang

Huazhong University of Science and Technology

Xuan Wang

Huazhong University of Science and Technology

\section{Research Article}

Keywords: ECM, matrisome, glioma, immune microenvironment

Posted Date: January 28th, 2022

DOI: https://doi.org/10.21203/rs.3.rs-1087869/v2

License: (c) (i) This work is licensed under a Creative Commons Attribution 4.0 International License. Read Full License 


\section{Abstract}

\section{Background}

The extracellular matrix (ECM) plays a vital role in the progression and metastasis of glioma and is an important part of the tumor microenvironment. However, there are few studies on the overall role of the ECM in the glioma immune microenvironment. This study aimed to analyze the prognosis of matrisomes in patients with glioma.

\section{Methods}

Overall, 676 glioma patients in The Cancer Genome Atlas (TCGA) database were divided into the low, moderate, and high immune infiltration groups. Immune-related matrisomes differentially expressed among the three groups were analyzed, and a risk signature was established. Eight immune-related matrisomes were screened, namely, LIF, LOX, MMP9, S100A4, SRPX2, SLIT1, SMOC1, and TIMP1. Kaplan Meier analysis, operating characteristic curve (ROC) analysis and nomogram were constructed to analyze the relationships between risk signatures and the prognosis of glioma patients.

\section{Results}

The risk signature was significantly correlated with the overall survival (OS) of glioma patients. Both highand low-risk signatures were also associated with some immune checkpoints. In addition, analysis of somatic mutations and anti-PD1/L1 immunotherapy responses in the high- and low-risk groups showed that the high-risk group had worse prognosis and a higher response to anti-PD1/L1 immunotherapy. qPCR and immunohistochemical analysis showed that LIF, LOX, MMP9, S100A4, SRPX2 and TIMP1 were highly expressed in glioma, while SLITI1 and SMOC1 were low expressed in glioma.

\section{Conclusions}

Matrisomes play a vital role in the complex immune microenvironment of gliomas. Our analysis of immune-related matrisomes can improve understanding of the characteristics of the glioma immune microenvironment and provide important clues for glioma immunotherapy in the future.

\section{Introduction}

Glioblastoma (GBM) is the most prevalent and aggressive malignant tumor of the central nervous system (CNS), accounting for $14.6 \%$ of all CNS tumors[1]. Latest data show that approximately 100,000 incident cases of GBMs are diagnosed annually. Gliomas are differentiated from glial cells and are histologically classified as astrocytoma, oligodendroglioma, oligodendrocytoma, and ependymoma[2]. GBM has an unfavorable prognosis, with a median survival time of only 14-17 months and an average 2- and 3-year survival rates of only $3.3 \%$ and $1.2 \%$, respectively[3]. Although surgery combined with radiotherapy and chemotherapy has achieved good results in clinical trials, the recurrence rate is still very high[4]. This poor prognosis of glioma is attributed to several factors, such as the unique location, high heterogeneity, and 
tumor immunosuppressive microenvironment[5]. Immunotherapy has unsatisfactory effects on glioma owing to the immune-cold phenotype and unique immune microenvironment[6]. Therefore, there is an urgent need to identify improved indicators for the immune microenvironment of glioma.

The extracellular matrix (ECM), an important component of an organism, is a complex scaffold network composed of cross-linked proteins such as collagen, non-collagen, elastin, proteoglycan, and aminoglycan that supports surrounding cells[7]. ECM can provide a tissue scaffold to maintain the morphology and integrity of organs and is very important in cell development[8]. ECM proteins contain abundant signal molecules that control cell growth, polarity, shape, migration, and metabolic activity[9]. The collection of ECM molecules and related proteins is called a matrisome[10].

ECM can also promote tumor growth, providing adhesion sites and cell signals to different cell groups, including cancer cells. ECM can also store many ECM-modifying enzymes, ECM binding growth factors, and other ECM-related proteins that assist with cell signaling of ECM proteins[11]. ECM is also involved in some pathological processes in the body, such as inflammation and tumors. Studies have shown that an increase in the ECM component is linked to tumor invasion and poor prognosis[12, 13]. The ECM plays a crucial role in tumor progression, invasion, and metastasis. For example, lysyl oxidase (LOX) is a monoamine oxidase associated with metastasis and adverse prognosis in gastric cancer[14]. Matrix metalloproteinase-9 (MMP9) can act on the degradation of ECM and vascular remodeling, thus promoting tumor invasion[15].

Matrisomes are also involved in glioma development. The proteolytic activity of ECM is associated with invasion and metastasis of gliomas[16]. The immune microenvironment of glioma is composed of glioma cells, immune cells, and the ECM. Studies have shown that the ECM is related to the glioma immune microenvironment[17]. Thus, there have been several studies on the effects of matrisomes on the glioma immune microenvironment. However, most of these studies were performed on individual glioma immune-related matrisomes rather than integral analysis.

Hence, an overall analysis of all potential immune-related matrisomes in gliomas is needed. In addition, because of the important prognostic influence of immune infiltration in gliomas, it is essential to clarify whether immune-related matrisomes have prognostic value. Thus, this study intended to analyze the expression profile of matrisomes in 667 patients with glioma, using the whole transcriptome dataset from The Cancer Genome Atlas (TCGA) database.

\section{Materials And Methods}

\subsection{Data sources and clinical samples}

Human matrisomes were collected from the Molecular Signatures Database V7.4. Meanwhile, the fragments per kilobase per million (FPKM) of transcriptome data, statistical data, and clinical features of patients with human low-grade glioma (LGG) and GBM were extracted from the Cancer Genome Atlas Database (TCGA, https://cancergenome.nih.gov/)[18]. Patient characteristics included the total number 
of patients ( $n=667)$, gender, age, grade, isocitrate dehydrogenase (IDH) status, Karnofsky performance score (KPS), chemotherapy, radiotherapy, and MGMT promoter methylation status. Verified FPKM transcriptome data and patient clinical characteristics $(n=970)$ were extracted from the Chinese Glioma Genome Atlas (CGGA) database (http://www.cgga.org.cn/)[19].

In addition, brain tissue samples of 26 patients with GBM and 18 patients with paracancerous lesions were obtained from Union Hospital, Tongji Medical College, Huazhong University of Science and Technology, Wuhan, China.

\subsection{Generation and verification of immune grouping}

We used single-sample gene set enrichment analysis (SSGSEA) to analyze the level of immune infiltration in glioma samples. The glioma patients were classified into three groups according to the level of immune cell infiltration using "hclust" (R package). To verify the correctness of clustering, immune cell expression in the three groups was calculated using the CIBERSORT deconvolution algorithm, and a heatmap was generated. The differences among the three groups were verified by stromal score, immune score, estimated score, tumor purity, human leukocyte antigen (HLA) expression level, and immune cell composition.

\subsection{Screening of immune-related matrisomes}

We divided the TCGA gene expression profile data of glioma patients into three groups: low, medium, and high immune cell infiltration. Taking $\left|\log _{2} \mathrm{FC}\right|>1, \mathrm{P}$ value $<0.05$ as the standard, the differentially expressed matrisomes between the low and moderate immune cell infiltration groups and between the moderate and high immune cell infiltration groups were analyzed using "edgeR" package. Using online Venn analysis, overlapping matrisomes between the above two groups of differentially expressed matrisomes were selected to screen immune-related matrisomes.

\subsection{Establishing a risk signature based on immune-related prognostic matrisomes}

The relationship between immune-related matrisome expression and overall survival (OS) of glioma patients was analyzed using univariate Cox regression. In univariate Cox analysis, a $P$-value $<0.05$ was considered statistically significant. Least absolute shrinkage and selection operator (LASSO) regression was used to screen the applicable combination of immune-related prognostic matrisomes, and a risk signature was constructed. The candidate genes were divided into two groups: risk type $(H R>1)$ and protective type $(0,<1)$. A prognostic risk score equation was then established based on the results of LASSO analysis, and a linear combination of expression level and regression coefficient weighting was 
used. The risk score equation was as follows: risk score $=$ matrisome $(1) \times \beta(1)$ expression + matrisome $(2) \times \beta(2)+\ldots \ldots .+$ Matrisome $n \times \beta(n)$.

Kaplan Meier (KM) curves and time-dependent receiver operating characteristic (ROC) curves were then generated based on the median risk score to verify the prognostic significance of risk signature. A total of 667 patients with glioma were divided into the high-risk and low-risk subgroups (Supplementary Table S1). The effectiveness of the risk signature was further verified using the CGGA dataset comprising 970 glioma patients (Supplementary Table S2).

\subsection{Establishing and assessing the nomogram}

To improve the clinical value of the risk signature in the clinic, we conducted univariate and multivariate Cox regression analyses to determine the relationship between factors (gender, age, grade, IDH status, $1 p / 19 q$ codeletion, Karnofsky performance score, MGMT promoter status, history of radiotherapy and chemotherapy, and risk score) and OS. The nomogram was established using the TCGA database and verified using the CGGA database. The prognostic predictive accuracy of the nomogram was demonstrated using calibration curves. These analyses were performed using the R package "rms." ROC curves were used to evaluate the prognostic capacity of the nomogram and other predictors (risk score, age, grade, IDH mutation status, and 1p/19q codeletion) for 1-, 3-, and 5-year OS of patients with glioma.

\subsection{Functional enrichment analysis}

To further explore the 8 immune-related matrisomes, we screened differentially expressed genes between the high- and low-risk subgroups using R software package "edgeR." The functional enrichment of the differentially expressed genes was then analyzed with the Gene Ontology (GO) enrichment analysis and Kyoto Encyclopedia of Genes and Genomes (KEGG) using the R software package "ggpolt2," with | log2fc $\mid>1$ and $P$ value $<0.05$ as the standard.

\subsection{Immunohistochemistry}

The paraffin-embedded tissues of 16 patients with GBM and 12 patients with paracancerous brain tissue were cut into $5 \mu \mathrm{m}$-thick sections and stained with hematoxylin. The sections were incubated with antileukemia inhibitory factor (LIF), LOX, MMP9, S100A4, SRPX2, SLIT1, SMOC1, and TIMP1 primary antibodies at $4^{\circ} \mathrm{C}$; washed with phosphate buffer three times; and incubated with secondary antibody at room temperature for $30 \mathrm{~min}$.

We used 3,3-diaminobenzidine (DAB; 1:50) as the chromogenic substrate and counterstained with hematoxylin[20]. The staining was evaluated by two independent pathologists who were blinded to the patients' clinical data. The tissue sections were scored according to the percentage of staining positive cells as follows: $0,0 \% ; 1,1-10 \% ; 2,11 \%-25 \% ; 3,26-50 \% ; 4,51-70 \%$; and $5,26-50 \%$. Meanwhile, staining 
intensity was scored as 0 for negative staining; 1, weak staining; 2, moderate staining; and 3, strong staining. The final staining score was calculated as the product of staining intensity and percentage of positive cells. The samples with a final staining score of $<3$ and $\geq 3$ were divided into the negative and positive expression groups, respectively.

\subsection{Real-time quantitative reverse transcriptase-polymerase chain reaction}

Total RNA was extracted from 10 glioma and 6 adjacent brain tissue samples using TRIzol reagent (Thermo Fisher Scientific). A reverse transcription kit (Vazyme, Wuhan, China) was used to reverse transcribe CDNA, and then real-time polymerase chain reaction (PCR) was performed with SYBR Green real-time PCR kit, with GAPDH as the internal reference control. The PCR cycle conditions were as follows: $95^{\circ} \mathrm{C}$ for $2 \mathrm{~min}$, then $94^{\circ} \mathrm{C}$ for $20 \mathrm{~s}, 58^{\circ} \mathrm{C}$ for $20 \mathrm{~s}$, and $72^{\circ} \mathrm{C}$ for $30 \mathrm{~s}$, for a total of 40 cycles. All RT-qPCR reactions were independently performed three times. The tissue samples and primer sequences of the matrisomes are shown in Supplementary Tables S3 and S4.

\subsection{Tumor mutation burden and anti-PD1/L1 therapy of risk groups}

Somatic mutation data were obtained from TCGA database. Somatic mutations mainly included frameshift mutations, non-synonymous mutations, non-silent mutations, frameshift mutations, and deletion mutations. The tumor mutation burden (TMB) as the number of somatic mutations per megabyte. We used the R package "maftools" to analyze the mutation profiles[21]. Meanwhile, tumor immune dysfunction and exclusion (TIDE; http://tide.dfci.harvard.edu) and immune cell abundance identifier (ImmuCellAl; http://bioinfo.life.hust.edu.cn/ImmuCellAl) algorithms were used to analyze the potential response to PD1/L1 therapy.

\subsection{Statistical analysis}

The ROC curve was generated to measure the accuracy of survival prediction, using the R package "survivaIROC." Student's t-test was used to compare the risk scores among subgroups based on clinical characteristics. The Wilcoxon test was used to compare the immune infiltration data between the risk groups. GSEA was used for the functional analysis[22]. All statistical analyses were performed using the $\mathrm{R}$ 4.0.2. Statistical significance was defined as a two-tailed P-value $<0.05$ ( $* \star \star, p<0.001$; $* \star, p<0.01$; and *, $p<0.05)$. All pictures were drawn using the $\mathrm{R}$ language.

\section{Results}




\subsection{Identification of immune-related matrisomes in glioma patients}

A total of 667 low-grade glioma (LGG) and GBM samples were extracted from TCGA database. The clinical characteristics of the patients are summarized in Table 1.

Of these, 269, 338, and 61 samples were categorized into the low infiltration (immunity_L), moderate infiltration (immunity_M), and high infiltration (immunity_H) groups, respectively, using the unsupervised hierarchical clustering algorithm (Figure 1A). As seen in Figure 1B-1D, there were notable differences in immune score, stromal score, and estimate score among the three groups, and the score increased gradually from the low immune group to the high immune infiltration group $(p<0.001)$. Tumor purity was also significantly different, increasing from the high infiltration group to the low infiltration group $(\mathrm{P}<$ 0.001).

In addition, HLA expression and the types of immune cell infiltration in the high infilitration group were higher than those in the other two groups $(P<0.001)$. For differentially expressed matrisomes, 119 were differentially expressed between the low and moderate infiltration group; of these 97 and 22 matrisomes were upregulated and downregulated, respectively. Meanwhile, 76 matriomes were differentially expressed between the moderate and high infiltration groups; of these, 46 and 30 were upregulated and downregulated, respectively (Figures $1 \mathrm{E}$ and $1 \mathrm{~F}$ ).

By intersecting with the above results, 36 differentially expressed matrisomes among the three groups were further screened by Venn analysis; among them, 28 and 8 were upregulated and downregulated, respectively.

Table 1

The clinical features of glioma patients in TCGA and CGGA database. 


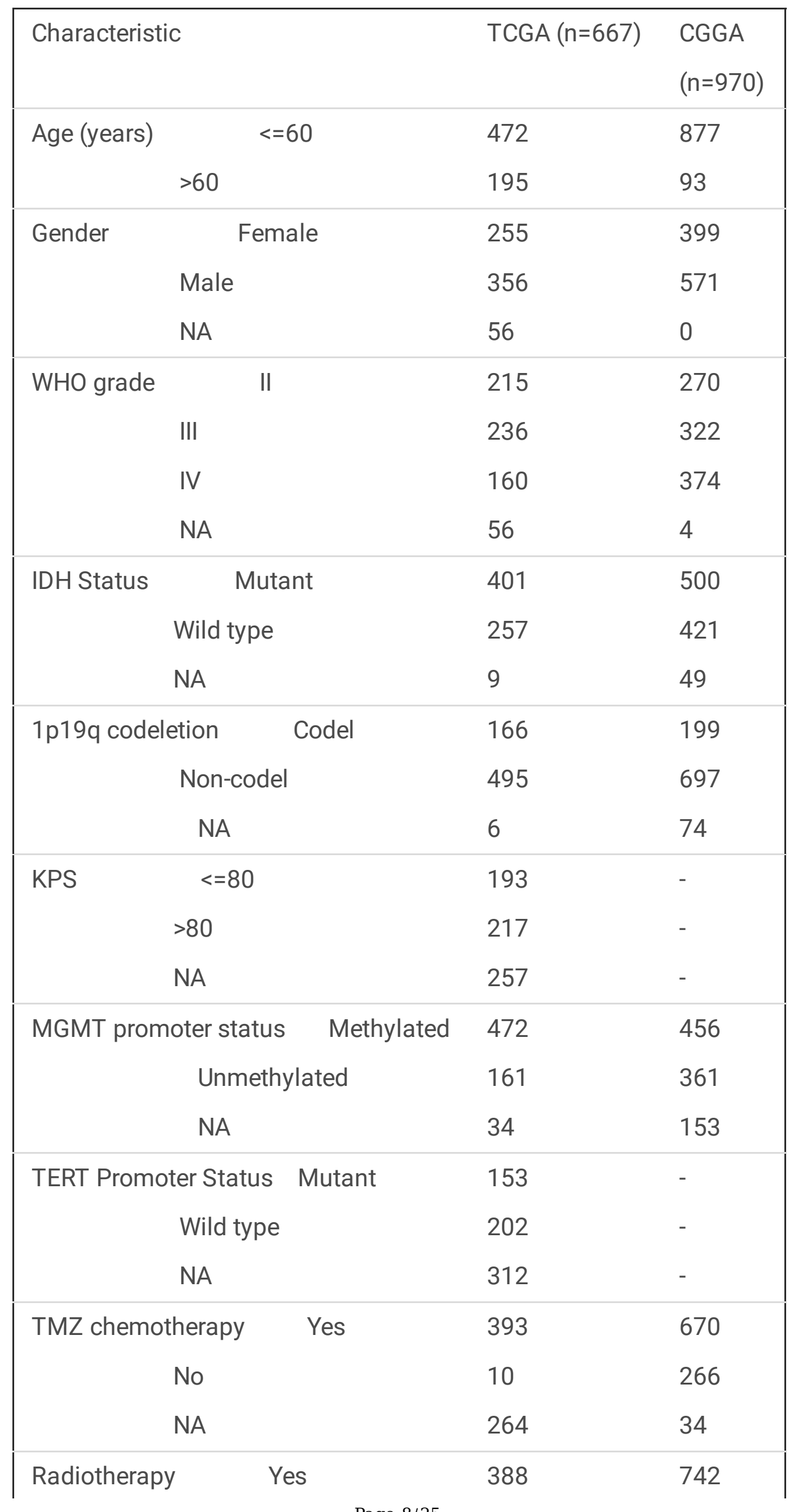




\begin{tabular}{|ccc|} 
No & 178 & 193 \\
NA & 101 & 35 \\
\hline KPS, Karnofsky Performance Score. & & \\
\hline
\end{tabular}

\subsection{Prognostic value of the risk signature}

In total, eight immune-related matrisomes were identified to have prognostic value, namely, LIF, LOX, MMP9, S100A4, SRPX2, SLITI1, SMOC1, and TIMP1 (Fig. 2A-2C). The survival analysis of their prognostic impact were shown in Supplementary Figure 1. According to median score, 315 and 352 patients were classified into the high- and low-risk groups. LIF, LOX, MMP9, S100A4, SRPX2, and TIMP1 were upregulated in the high-risk group, whereas SLIT1 and SMOC1 were downregulated in the low-risk group. The OS rate was significantly worse in the high-risk group than in the low-risk group $(P<0.001)$ (Fig. 2D), demonstrating that the risk score had good prognostic value.

The risk curve and scatter plot showed that the mortality rate was higher in the high-risk group than in the low-risk group (Fig. 2E). On ROC curve analysis to verify the predictive significance of the risk signatures, the AUCs for predicting $1-, 3$-, and 5-year survival rates were $0.885,0.895$, and 0.844 , respectively, in TCGA database (Fig. 2F). Further verification using the CGGA database showed AUCs of 0.813, 0.814, and 0.803 for predicting the 1-, 3-, and 5-year survival rates, respectively (Fig. 2G-2I).

\section{3. Association between risk signature and clinical parameters}

The heatmap showed the relationship between high- and low-risk groups and clinical features of glioma patients, such as gender, age, WHO grade, IDH mutation status, KPS, 1p/19q codeletion, TERT promoter status, and MGMT promoter methylation status, in TCGA database (Fig. 3A). There were more elderly patients in the high-risk group, and patients aged $<60$ years had significantly lower risks of mortality than did patients aged $>60$ years $(P<0.001)$ (Fig. 3B). Patients with KPS $>80$ had a significantly lower risk score than those with a KPS $<80(P<0.001)$ (Fig. 3G). There was no significant difference in the distribution of gender between the high-risk and low-risk groups (Fig. $3 \mathrm{C}$ ).

Meanwhile, there were more high WHO grade patients in the high-risk group and more low WHO grade patients in the low-risk group $(\mathrm{P}<0.001)$ (Fig. 3D). For IDH mutation status, IDH wild-type patients tended to be included in the high-risk group, while IDH-mutant patients tended to belong to the low-risk group $(\mathrm{P}<$ 0.001) (Fig. 3E). In addition, 1p/19q non-codeletion and MGMT promoter unmethylation were more frequent in the high-risk group $(\mathrm{P}<0.001)$ (Figures $3 \mathrm{~F}$ and $3 \mathrm{H})$. For TERT promoter status, patients with TERT promoter wild type had lower risk score than did patients with TERT promoter mutations $(P<0.001)$ (Fig. 3I). The clinical characteristics of glioma patients in the high- and low-risk groups in the CGGA database are shown in Supplementary Figure 2. 


\subsection{Nomogram performance for predicting 1-, 3- and 5-year survival rates}

The results of univariate and multivariate Cox analyses showed that the risk signature can be used as an independent prognostic factor in patients with glioma (Figure 4A and Fig. 4B). For practical application, a nomogram was established using the TCGA database to predict the 1-, 3-, and 5-year OS (Figure 4I), and the predictive performance was verified in the CGGA database (Supplementary Figure 3). The predictors included risk score, age, gender, IDH mutation, and 1p/19q codeletion status. The actual probabilities of 1-, 3-, and 5-year OS in the TCGA cohort (Fig. 4C-4E) and the CGGA cohort (Fig. S3C-S3E) were consistent with those predicted by the nomogram.

In addition, time-dependent ROC curves to evaluate the predictive capability of the nomogram and other predictors (risk score, age, grade, IDH status, and 1p/19q codeletion) showed AUCs of $0.890,0.942$, and 0.906 for TCGA (Fig. 4F-H), respectively, and $0.788,0.842$, and 0.843 , for the CGGA database (Fig. S3F-H), respectively. These results support that the nomogram is more reliable and accurate predictor of prognosis.

\subsection{Correlation between risk signature and immune infiltration of glioma microenvironment}

GSEA of high- and low-risk groups (Figure $5 \mathrm{~A}$ ) showed that in the $\mathrm{GO}$ signaling pathway, biological processes such as ECM tissue, leukocyte chemotaxis, myeloid leukocyte migration, and cytokine activity, were significantly related to the signature. For the KEGG signaling pathway, immune-related pathways and proliferation and migration-related pathways were significantly related to the signature. Immune-related pathways included viral protein cytokine, cytokine-cytokine receptor interaction, complement and coagulation cascade reaction, tumor necrosis factor signaling pathway, IL17 signaling pathway, toll-like receptor signaling pathway, chemokine signaling pathway, and transforming growth factor $\beta$ signaling pathways. Proliferation and migration-related pathways included the PI3K Akt and Wnt signaling pathways (Fig. 5B).

Given that the high- and low-risk groups were related to immune-related pathways, we explored the difference in immune cell infiltration between the two groups. There were significant differences in the levels of immune cells including T cells CD8, T cells CD4 memory resting, T cells CD4 memory activated, T cells regulatory (Tregs), $\mathrm{T}$ cells gamma delta, monocytes, macrophages $\mathrm{M} 0$, macrophages $\mathrm{M} 1$, macrophages M2, activated mast cells, activated NK cells, and neutrophils showed significant between the high- and low-risk groups $(P<0.05)$. Analysis of the correlation between risk scores and immune checkpoint expression (Supplementary Table S5) showed that the number of immune checkpoints, such as CD276, CD274, CTLA4, LAIR1, LILRA5, CD70, and LAG3, was positively related to the risk score. This indicated an immunosuppressive microenvironment in the high-risk glioma patients. 


\subsection{Association between tumor mutation burden and treatment response to anti-PD1/L1 therapy according to the risk signature}

As shown in Figure 6A-6B, we selected the top 20 significant mutant genes for analysis. The tumor mutation load was higher in the low-risk group than in the high-risk group. Mutant genes accounted for $99.38 \%$ and $89.47 \%$ of genes in the low- and high risk groups, respectively. Among them, the mutation frequencies of IDH1 and ATRX mutations were $46 \%$ and $28 \%$ in the high-risk group and were $93 \%$ and $39 \%$ in the low-risk group, respectively. This indicated that the high-risk group had worse prognosis. Meanwhile, the mutation frequencies of the oncogenes PTEN and EGFR were higher in the high-risk group than in the low-risk group ( $11 \%$ and $18 \%$ vs $2 \%$ and $0 \%$ ), indicating more aggressive disease in the highrisk group.

Analysis of the predictive value of immune-related matrisome risk signatures for treatment response to anti-PD1/L1 immunotherapy showed that in TIDE, 73\% and $61 \%$ of patients in the high- and low-risk groups responded to PD1/L1 immunotherapy, respectively (Figure 6D). In immuCellAl, 49\% and 32\% of patients in the high- and low-risk groups responded to PD1/L1 immunotherapy, respectively (Figure 6C).

\subsection{Expression of the eight immune-related matrisomes}

We verified the mRNA and protein levels of the eight immune-related matrisomes in brain tissue samples of 26 glioma patients and 18 patients with paracancerous lesions. Quantitative PCR and immunohistochemical analysis showed that LIF, LOX, MMP9, S100A4, SRPX2, and TIMP1 were expressed at high levels in glioma, whereas SLITI1 and SMOC1 were expressed at low levels, consistent with our previous results (Figure 7).

\section{Discussion}

Glioma is the most malignant tumor in the brain[23]. Despite several treatment options, including surgery, targeted radiotherapy, and chemotherapy, most patients with GBM die within 2 years[24]. Immunotherapy has made great progress in the treatment of hematological diseases such as lymphoma and leukemia[25, 26]. The main treatment strategies include immune checkpoint inhibitors, monoclonal antibodies, and cell therapy[27]. However, immunotherapy has unsatisfactory benefits for glioma, with patients still having low survival rate possibly owing to the unique immune microenvironment of gliomas[28].

GBM is characterized by remarkably unique location, high heterogeneity, and an "immune-cold" phenotype, promoting an immunosuppressive microenvironment[6]. There are only few tumor-infiltrating lymphocytes (TILs) in the glioma microenvironment, and they often show a state of exhaustion[29, 30]. Further, glioma cells can produce 2,3-dioxygenase to promote the accumulation of regulatory $T$ cells 
(Tregs), which can inhibit the function of T cells[31]. Moreover, M2 macrophages secrete proangiogenic factors and immunosuppressive cytokines, which can promote cancer progression. The abundance of immunosuppressive cells in the glioma microenvironment makes it challenging to overcome such immunosuppressive microenvironment[32,33]. Therefore, it is important to explore the components of the glioma microenvironment.

$\mathrm{ECM}$, an important component of the tumor microenvironment, can promote the formation and invasion of tumor[34]. The interaction of immune and tumor cells with ECM remodeling may affect tumor metastasis and therapeutic effect[35]. Considering the increasing importance of the role of matrisomes in tumors and their immune microenvironment, we constructed a risk signature using eight immune-related matrisomes, namely, LIF, LOX, MMP9, S100A4, SRPX2, SLIT1, SMOC1, and TIMP1, to predict the survival and prognosis of glioma patients. LIF can promote neural stem cell self-renewal in the brain[36]. It can also mediate tumor growth factor- $\beta$ to induce self-renewal of glioma-initiating cells (GICs)and prevent their differentiation[37].

LOX is an independent prognostic factor in patients with LGG, and high LOX expression is related to poor OS and response to targeted molecular therapy in patients with LGG[38]. MMP9 is associated with unfavorable prognosis of gliomas and is positively correlated with the grade of primary and recurrent gliomas[39]. S100A4 is a new marker and regulatory factor of glioma stem cells and a molecular chain of mesenchymal transition and stemness of GBM[40]. SRPX2 can promote epithelial-mesenchymal transformation in GBM and is also related to temozolomide resistance[41]. High levels of TIMP1 are associated with poor prognosis of GBM. In glioma cells, TIMP1 knockdown can delay tumor growth[42, 43].

$\mathrm{KM}$ curves, time-varying ROC curves, and a nomogram were used to verify the relation between the risk signature and clinical characteristics and prognosis of glioma patients. The functional enrichment pathway showed that the risk signature was related to tumor immunity, proliferation, and migration. Analysis of immune infiltration showed significant differences in the proportion of immune cells between the high- and low-risk groups. Furthermore, some immune checkpoints, such as CD274 and CTLA4, are related to the risk signature. Therefore, it is necessary to establish predictors that can reflect the somatic mutation burdens in the tumor microenvironment and patient prognosis. This will be helpful in understanding the composition of the tumor microenvironment and predicting response to immunotherapy.

We also found that the high-risk group was more sensitive to anti-PD1/L1 immunotherapy than is the low-risk group, possibly due to the higher expression of immune checkpoints in the high-risk group. The eight immune-related matrisomes identified may potentially help in the diagnosis and immunotherapy of glioma.

\section{Conclusions}


We screened and verified eight immune-related matrisomes and established a risk signature. The results showed that the risk signatures were significantly related to the prognosis of glioma patients and immune infiltration of the tumor microenvironment. Thus, they can serve as an indicator to predict the prognosis and immunotherapy response of glioma patients.

\section{Declarations}

\section{Author Contributions:}

Conceptualization, Xiaobing Jiang and Xuan Wang; methodology, Minjie Wang; software, Hao Yu; validation, Minjie Wang; formal analysis, Hao Yu.; data curation, Minjie Wang; writing-original draft preparation, Hao Yu.; writing-review and editing, Minjie Wang; visualization, Hao Yu; project administration, Xiaobing Jiang. All authors have read and agreed to the published version of the manuscript.

\section{Funding:}

This research was funded by NATIONAL NATURAL SCIENCE FOUNDATION OF CHINA, grant number 81974390.

\section{Ethics Statement:}

The study was conducted according to the guidelines of the Declaration of Helsinki, and approved by the Ethics Committee of TONGJI MEDICAL COLLEGE, HUAZHONG UNIVERSITY OF SCIENCE AND TECHNOLOGY (protocol code: [2019]IEC(S742). date of approval: MAR $4^{\text {th }} 2019$ ).

\section{Informed Consent Statement:}

Informed consent was obtained from all subjects involved in the study.

\section{Data Availability Statement:}

All RNA-seq files are available from the TCGA database (https://cancergenome.nih.gov/) and GGA (http://www.cgga.org.cn/) database within the article.

\section{Acknowledgments:}

We would like to thank all the people who helped us in the current study. 


\section{Conflicts of Interest:}

The authors declare no conflict of interest.

\section{References}

1. Omuro A, DeAngelis LM. Glioblastoma and other malignant gliomas: a clinical review. Jama. 2013;310(17):1842-50.

2. Ferris SP, Hofmann JW, Solomon DA, Perry A. Characterization of gliomas: from morphology to molecules. Virchows Archiv : an international journal of pathology. 2017;471(2):257-69.

3. Reifenberger G, Wirsching HG, Knobbe-Thomsen CB, Weller M. Advances in the molecular genetics of gliomas - implications for classification and therapy. Nature reviews Clinical oncology. 2017;14(7):434-52.

4. Stupp R, Hegi ME, Neyns B, Goldbrunner R, Schlegel U, Clement PM, Grabenbauer GG, Ochsenbein AF, Simon M, Dietrich PY, Pietsch T, Hicking C, Tonn JC, Diserens AC, Pica A, Hermisson M, Krueger S, Picard M, Weller M. Phase I/lla study of cilengitide and temozolomide with concomitant radiotherapy followed by cilengitide and temozolomide maintenance therapy in patients with newly diagnosed glioblastoma. Journal of clinical oncology : official journal of the American Society of Clinical Oncology. 2010;28(16):2712-8.

5. Gudbergsson JM, Christensen E, Kostrikov S, Moos T, Duroux M, Kjær A, Johnsen KB, Andresen TL. Conventional Treatment of Glioblastoma Reveals Persistent CD44(+) Subpopulations. Molecular neurobiology. 2020;57(9):3943-55.

6. Wang Z, Guo X, Gao L, Wang Y, Guo Y, Xing B, Ma W. Classification of pediatric gliomas based on immunological profiling: implications for immunotherapy strategies. Molecular therapy oncolytics. 2021;20:34-47.

7. Naba A, Clauser KR, Hoersch S, Liu H, Carr SA, Hynes RO. The matrisome: in silico definition and in vivo characterization by proteomics of normal and tumor extracellular matrices. Molecular \& cellular proteomics : MCP. 2012;11(4):M111.014647.

8. Socovich AM, Naba A. The cancer matrisome: From comprehensive characterization to biomarker discovery. Seminars in cell \& developmental biology. 2019;89:157-66.

9. Hynes RO, Naba A. Overview of the matrisome-an inventory of extracellular matrix constituents and functions. Cold Spring Harbor perspectives in biology. 2012;4(1):a004903.

10. Rafaeva M, Erler JT. Framing cancer progression: influence of the organ- and tumour-specific matrisome. The FEBS journal. 2020;287(8):1454-77.

11. Tian C, Öhlund D, Rickelt S, Lidström T, Huang Y, Hao L, Zhao RT, Franklin O, Bhatia SN, Tuveson DA, Hynes RO. Cancer Cell-Derived Matrisome Proteins Promote Metastasis in Pancreatic Ductal Adenocarcinoma. Cancer research. 2020;80(7):1461-74. 
12. Nelson CM, Bissell MJ. Of extracellular matrix, scaffolds, and signaling: tissue architecture regulates development, homeostasis, and cancer. Annual review of cell and developmental biology. 2006;22:287-309.

13. Bateman JF, Boot-Handford RP, Lamandé SR. Genetic diseases of connective tissues: cellular and extracellular effects of ECM mutations. Nature reviews Genetics. 2009;10(3):173-83.

14. Zhao L, Niu H, Liu Y, Wang L, Zhang N, Zhang G, Liu R, Han M. LOX inhibition downregulates MMP-2 and MMP-9 in gastric cancer tissues and cells. Journal of Cancer. 2019;10(26):6481-90.

15. Cabral-Pacheco GA, Garza-Veloz I, Castruita-De la Rosa C, Ramirez-Acuña JM, Perez-Romero BA, Guerrero-Rodriguez JF, Martinez-Avila N, Martinez-Fierro ML. The Roles of Matrix Metalloproteinases and Their Inhibitors in Human Diseases. International journal of molecular sciences. 2020;21(24).

16. VanMeter TE, Rooprai HK, Kibble MM, Fillmore HL, Broaddus WC, Pilkington GJ. The role of matrix metalloproteinase genes in glioma invasion: co-dependent and interactive proteolysis. Journal of neuro-oncology. 2001;53(2):213-35.

17. Strepkos D, Markouli M, Klonou A, Piperi C, Papavassiliou AG. Insights in the immunobiology of glioblastoma. Journal of molecular medicine (Berlin, Germany). 2020;98(1):1-10.

18. Jia D, Li S, Li D, Xue H, Yang D, Liu Y. Mining TCGA database for genes of prognostic value in glioblastoma microenvironment. Aging. 2018;10(4):592-605.

19. Zhao Z, Zhang KN, Wang Q, Li G, Zeng F, Zhang Y, Wu F, Chai R, Wang Z, Zhang C, Zhang W, Bao Z, Jiang T. Chinese Glioma Genome Atlas (CGGA): A Comprehensive Resource with Functional Genomic Data from Chinese Glioma Patients. Genomics, proteomics \& bioinformatics. 2021.

20. Goldsmith JD, Fitzgibbons PL, Swanson PE. Principles of Analytic Validation of Clinical Immunohistochemistry Assays. Advances in anatomic pathology. 2015;22(6):384-7.

21. Mayakonda A, Lin DC, Assenov Y, Plass C, Koeffler HP. Maftools: efficient and comprehensive analysis of somatic variants in cancer. Genome research. 2018;28(11):1747-56.

22. Subramanian A, Tamayo P, Mootha VK, Mukherjee S, Ebert BL, Gillette MA, Paulovich A, Pomeroy SL, Golub TR, Lander ES, Mesirov JP. Gene set enrichment analysis: a knowledge-based approach for interpreting genome-wide expression profiles. Proceedings of the National Academy of Sciences of the United States of America. 2005;102(43):15545-50.

23. Ostrom QT, Bauchet L, Davis FG, Deltour I, Fisher JL, Langer CE, Pekmezci M, Schwartzbaum JA, Turner MC, Walsh KM, Wrensch MR, Barnholtz-Sloan JS. The epidemiology of glioma in adults: a "state of the science" review. Neuro-oncology. 2014;16(7):896-913.

24. Bilmin K, Kujawska T, Grieb P. Sonodynamic Therapy for Gliomas. Perspectives and Prospects of Selective Sonosensitization of Glioma Cells. Cells. 2019;8(11).

25. Höpken UE, Rehm A. Targeting the Tumor Microenvironment of Leukemia and Lymphoma. Trends in cancer. 2019;5(6):351-64.

26. Aldoss I, Forman SJ. How I treat adults with advanced acute lymphoblastic leukemia eligible for CD19-targeted immunotherapy. Blood. 2020;135(11):804-13. 
27. Riley RS, June $\mathrm{CH}$, Langer R, Mitchell MJ. Delivery technologies for cancer immunotherapy. Nature reviews Drug discovery. 2019;18(3):175-96.

28. Pinton L, Masetto E, Vettore M, Solito S, Magri S, D'Andolfi M, Del Bianco P, Lollo G, Benoit JP, Okada H, Diaz A, Della Puppa A, Mandruzzato S. The immune suppressive microenvironment of human gliomas depends on the accumulation of bone marrow-derived macrophages in the center of the lesion. Journal for immunotherapy of cancer. 2019;7(1):58.

29. Woroniecka K, Chongsathidkiet P, Rhodin K, Kemeny H, Dechant C, Farber SH, Elsamadicy AA, Cui X, Koyama S, Jackson C, Hansen LJ, Johanns TM, Sanchez-Perez L, Chandramohan V, Yu YA, Bigner DD, Giles A, Healy P, Dranoff G, Weinhold KJ, Dunn GP, Fecci PE. T-Cell Exhaustion Signatures Vary with Tumor Type and Are Severe in Glioblastoma. Clinical cancer research : an official journal of the American Association for Cancer Research. 2018;24(17):4175-86.

30. Klemm F, Maas RR, Bowman RL, Kornete M, Soukup K, Nassiri S, Brouland JP, lacobuzio-Donahue CA, Brennan C, Tabar V, Gutin PH, Daniel RT, Hegi ME, Joyce JA. Interrogation of the Microenvironmental Landscape in Brain Tumors Reveals Disease-Specific Alterations of Immune Cells. Cell. 2020;181(7):1643-60.e17.

31. See AP, Parker JJ, Waziri A. The role of regulatory $T$ cells and microglia in glioblastoma-associated immunosuppression. Journal of neuro-oncology. 2015;123(3):405-12.

32. Murdoch C, Muthana M, Coffelt SB, Lewis CE. The role of myeloid cells in the promotion of tumour angiogenesis. Nature reviews Cancer. 2008;8(8):618-31.

33. Cui X, Morales RT, Qian W, Wang H, Gagner JP, Dolgalev I, Placantonakis D, Zagzag D, Cimmino L, Snuderl M, Lam RHW, Chen W. Hacking macrophage-associated immunosuppression for regulating glioblastoma angiogenesis. Biomaterials. 2018;161:164-78.

34. Bonnans C, Chou J, Werb Z. Remodelling the extracellular matrix in development and disease. Nature reviews Molecular cell biology. 2014;15(12):786-801.

35. Kai F, Drain AP, Weaver VM. The Extracellular Matrix Modulates the Metastatic Journey. Developmental cell. 2019;49(3):332-46.

36. Ying QL, Nichols J, Chambers I, Smith A. BMP induction of Id proteins suppresses differentiation and sustains embryonic stem cell self-renewal in collaboration with STAT3. Cell. 2003;115(3):281-92.

37. Peñuelas S, Anido J, Prieto-Sánchez RM, Folch G, Barba I, Cuartas I, García-Dorado D, Poca MA, Sahuquillo J, Baselga J, Seoane J. TGF-beta increases glioma-initiating cell self-renewal through the induction of LIF in human glioblastoma. Cancer cell. 2009;15(4):315-27.

38. Huang SP, Chiou J, Jan YH, Lai TC, Yu YL, Hsiao M, Lin YF. Over-expression of lysyl oxidase is associated with poor prognosis and response to therapy of patients with lower grade gliomas. Biochemical and biophysical research communications. 2018;501(3):619-27.

39. Zhou W, Yu X, Sun S, Zhang X, Yang W, Zhang J, Zhang X, Jiang Z. Increased expression of MMP-2 and MMP-9 indicates poor prognosis in glioma recurrence. Biomedicine \& pharmacotherapy $=$ Biomedecine \& pharmacotherapie. 2019;118:109369. 
40. Chow KH, Park HJ, George J, Yamamoto K, Gallup AD, Graber JH, Chen Y, Jiang W, Steindler DA, Neilson EG, Kim BYS, Yun K. S100A4 Is a Biomarker and Regulator of Glioma Stem Cells That Is Critical for Mesenchymal Transition in Glioblastoma. Cancer research. 2017;77(19):5360-73.

41. Tang H, Zhao J, Zhang L, Zhao J, Zhuang Y, Liang P. SRPX2 Enhances the Epithelial-Mesenchymal Transition and Temozolomide Resistance in Glioblastoma Cells. Cellular and molecular neurobiology. 2016;36(7):1067-76.

42. Chen PY, Li XD, Ma WN, Li H, Li MM, Yang XY, Li SY. Comprehensive Transcriptomic Analysis and Experimental Validation Identify IncRNA HOXA-AS2/miR-184/COL6A2 as the Critical ceRNA Regulation Involved in Low-Grade Glioma Recurrence. OncoTargets and therapy. 2020;13:4999-5016.

43. Aaberg-Jessen C, Sørensen MD, Matos A, Moreira JM, Brünner N, Knudsen A, Kristensen BW. Coexpression of TIMP-1 and its cell surface binding partner CD63 in glioblastomas. BMC cancer. 2018;18(1):270.

\section{Figures}


A

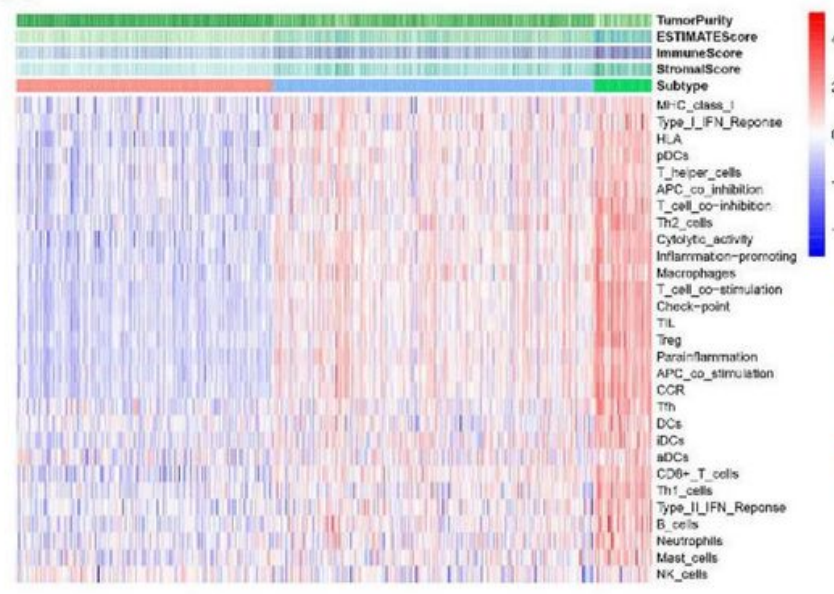

C

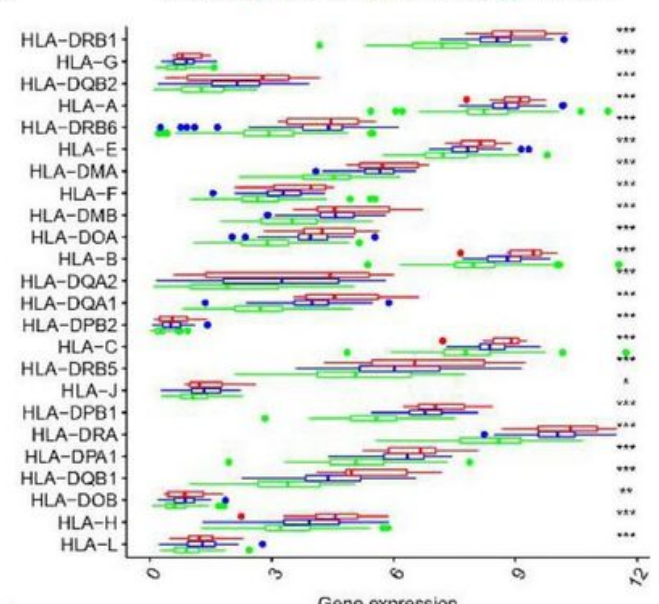

$\mathrm{E}$

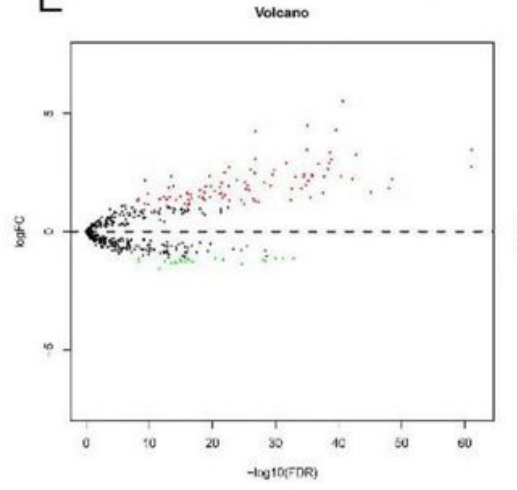

Volcano

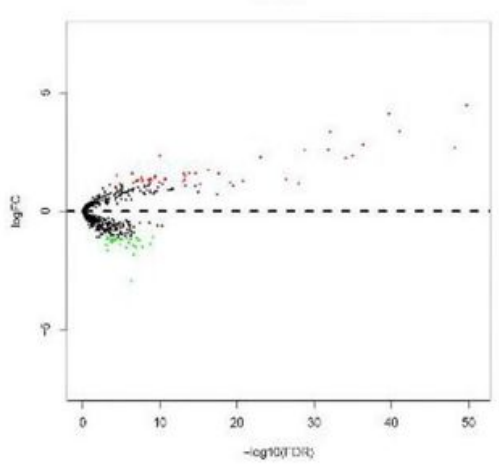

B

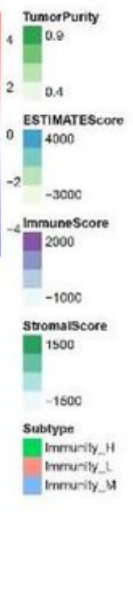

D

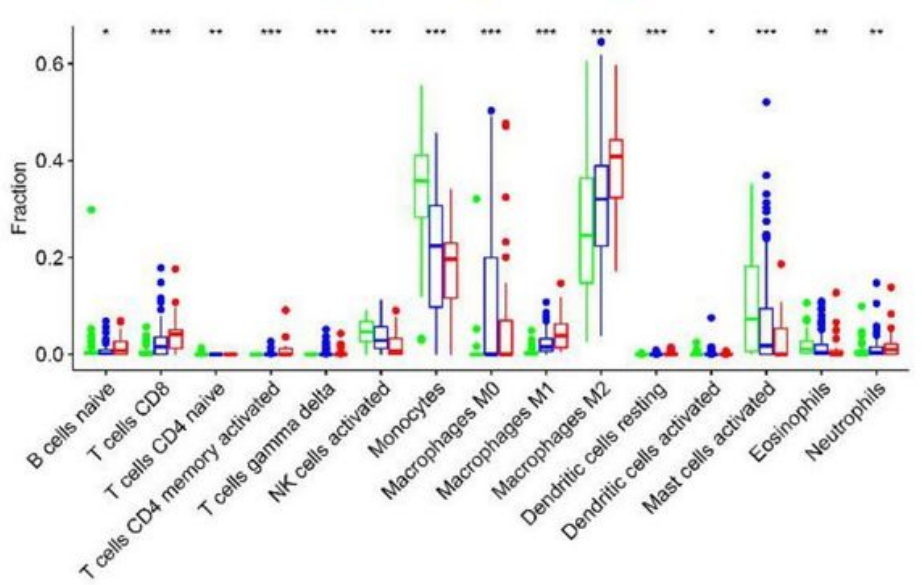

$\mathrm{F}$

Up

Down

Low vs. Medium
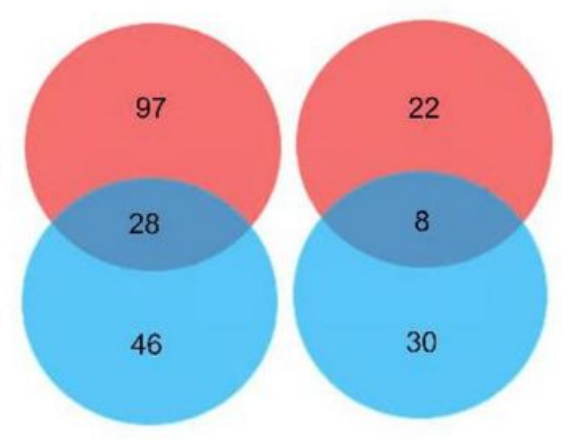

\section{Figure 1}

Characteristics of immune grouping and screening of immune-related matrisomes. (A) A total of 667 glioma samples were divided into three groups based on immune cell infiltration: low infiltration group (immunity_L, $n=269$ ), medium infiltration group (immunity_M, $\mathrm{n}=338$ ) and high infiltration group (immunity_H, $n=61$ ). (B) ESTIMATE Score, Immune Score, Stromal Score and Tumor Purity of immune groups. (C) HLA expression of the three immune groups. (D) The immune infiltration of different groups. 
$(E)$ and $(F)$ are differentially expressed matrisomes and intersection Venn diagrams of the three immune groups.
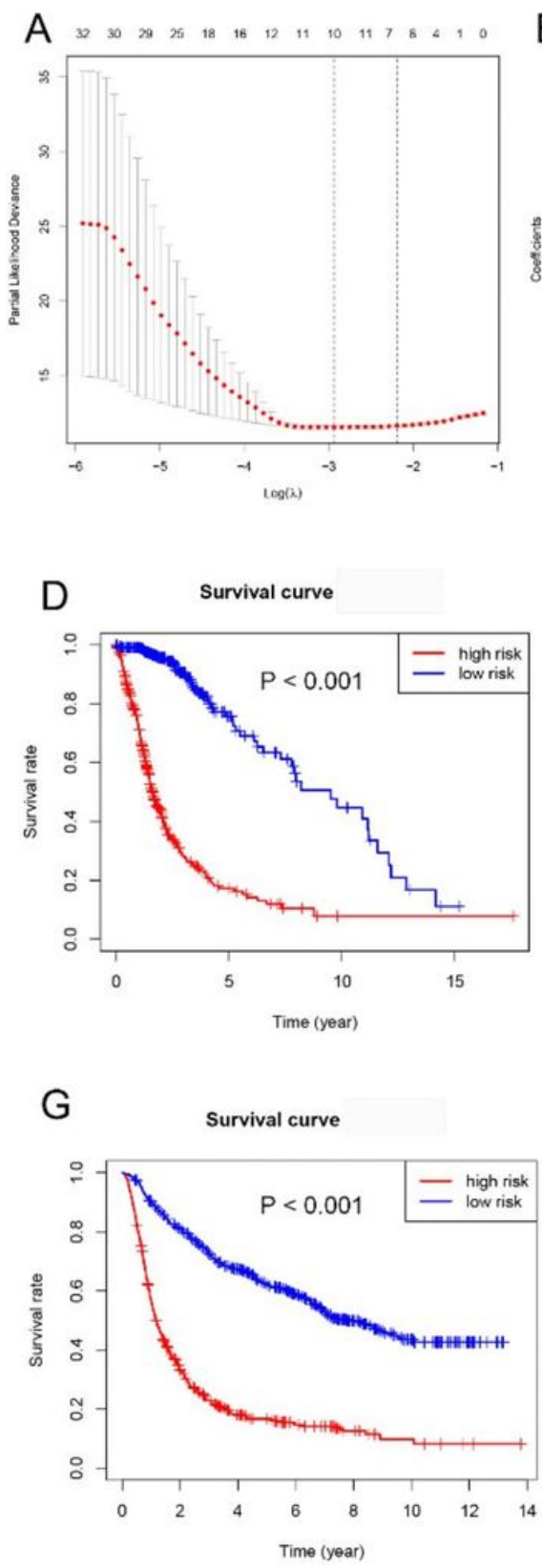
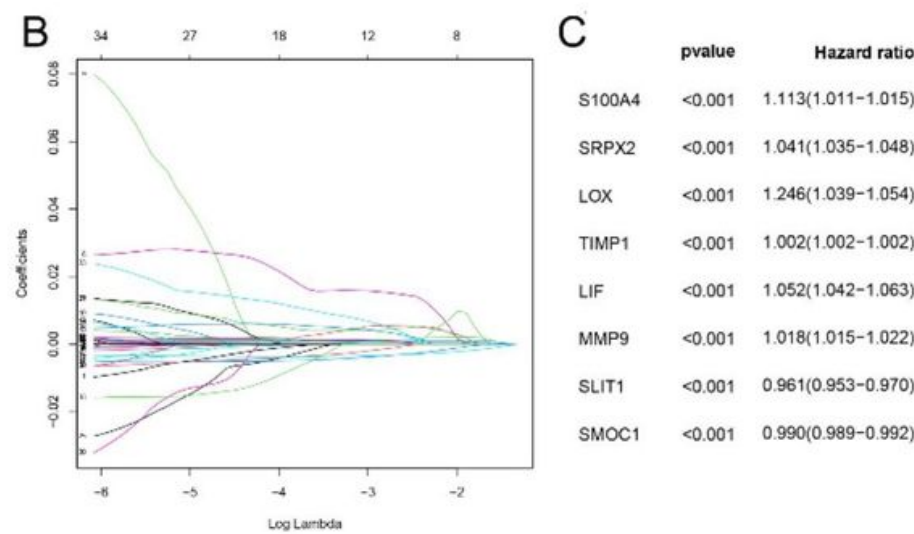

$\mathrm{E}$

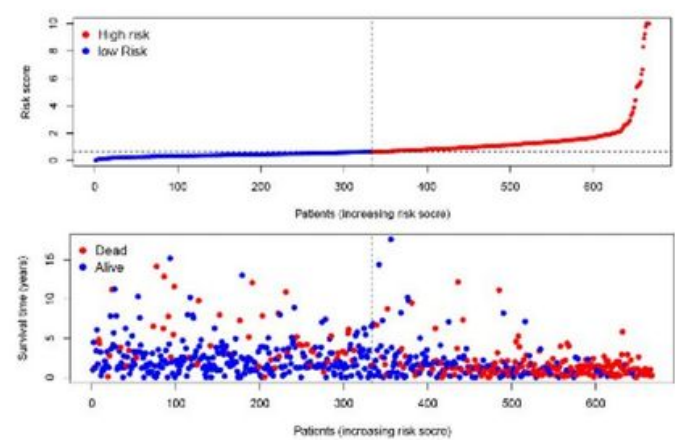

$\mathrm{H}$

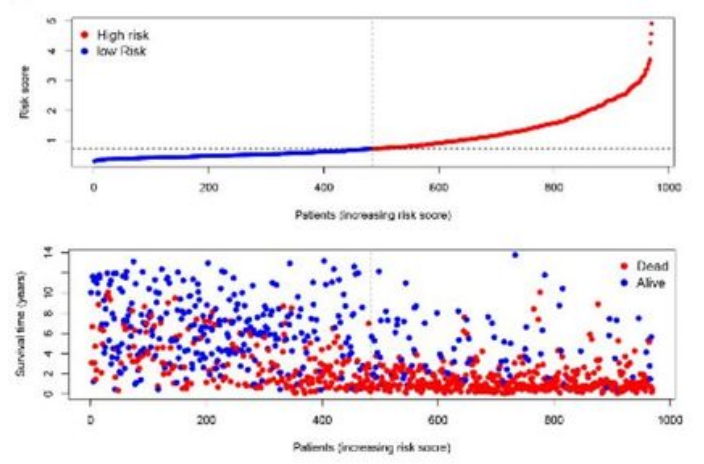

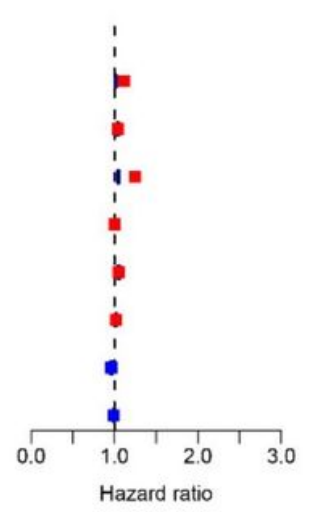

$\mathrm{F}$
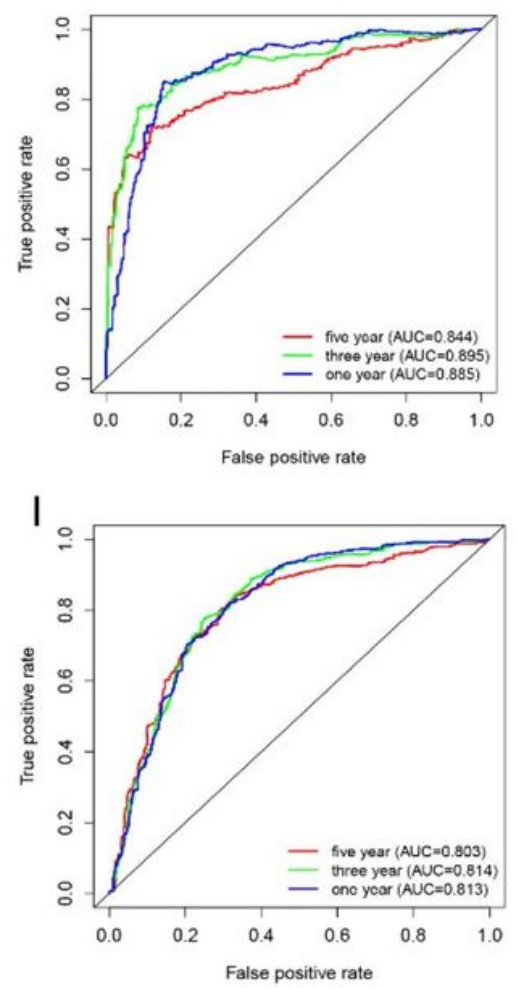

\section{Figure 2}

Establish and analyze immune-related matrisomes risk signatures for the prognosis of glioma. $(A)$ and (B) LASSO regression analysis verified the prognosis of 8 immune-related matrisomes. (C) Univariable Cox regression of 8 immune-related matrisomes in TCGA database. (D) The Kaplan Meier (KM) curve showed that the overall survival rate in the high-risk group was worse than that in the low-risk group in TCGA database. (E) The risk curve and scatter plot of high and low group in TCGA database. (F) The AUCs of the 1-year, 3-year and 5-year survival rates in TCGA database. (G) The Kaplan Meier (KM) curve indicated that the overall survival rate in the high-risk group was worse than that in the low-risk group in 
CGGA database. $(\mathrm{H})$ The risk curve and scatter plot of high and low group in CGGA database. (I) The AUCs of the 1-year, 3-year and 5-year survival rates in CGGA database.

A
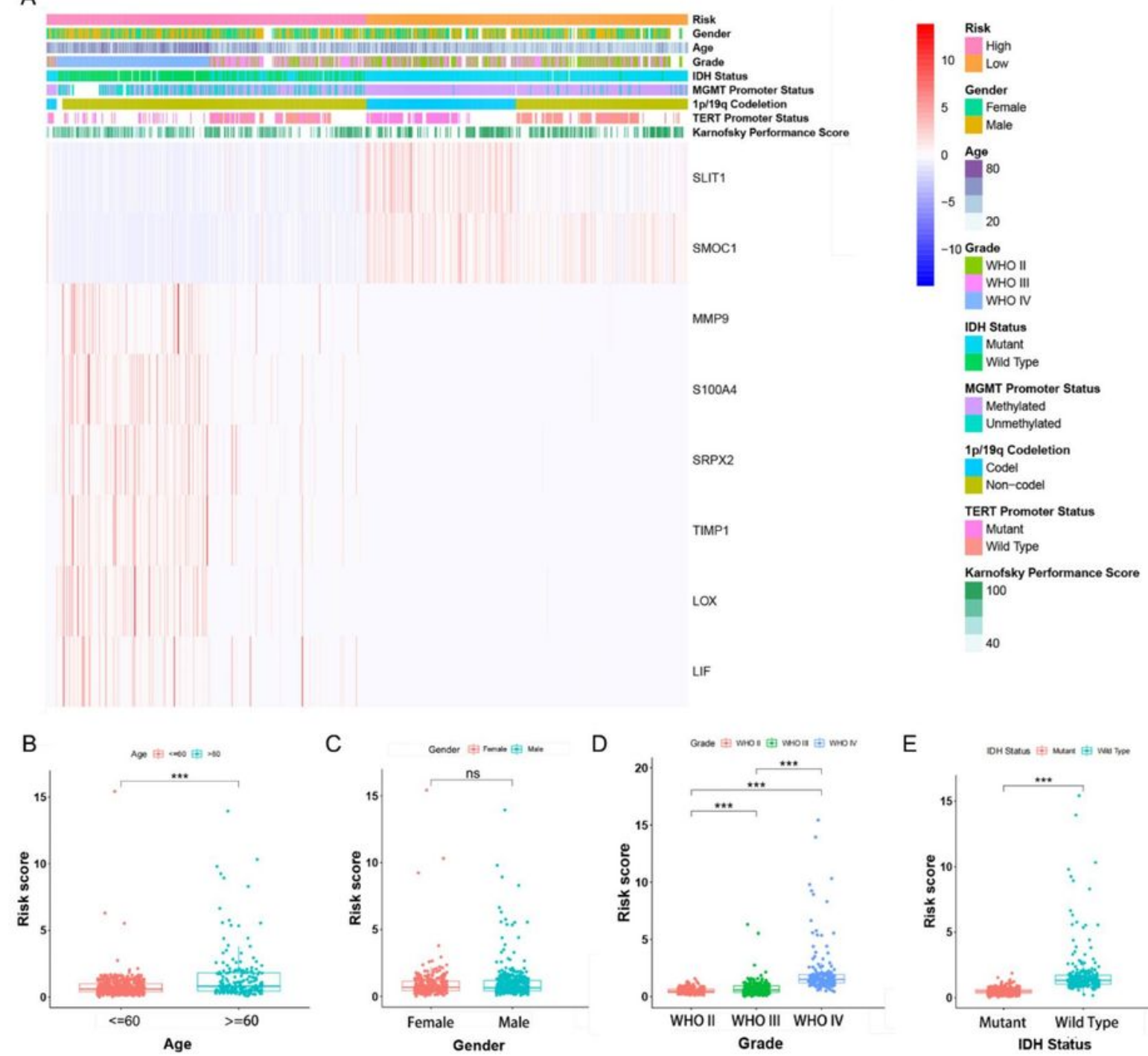

C
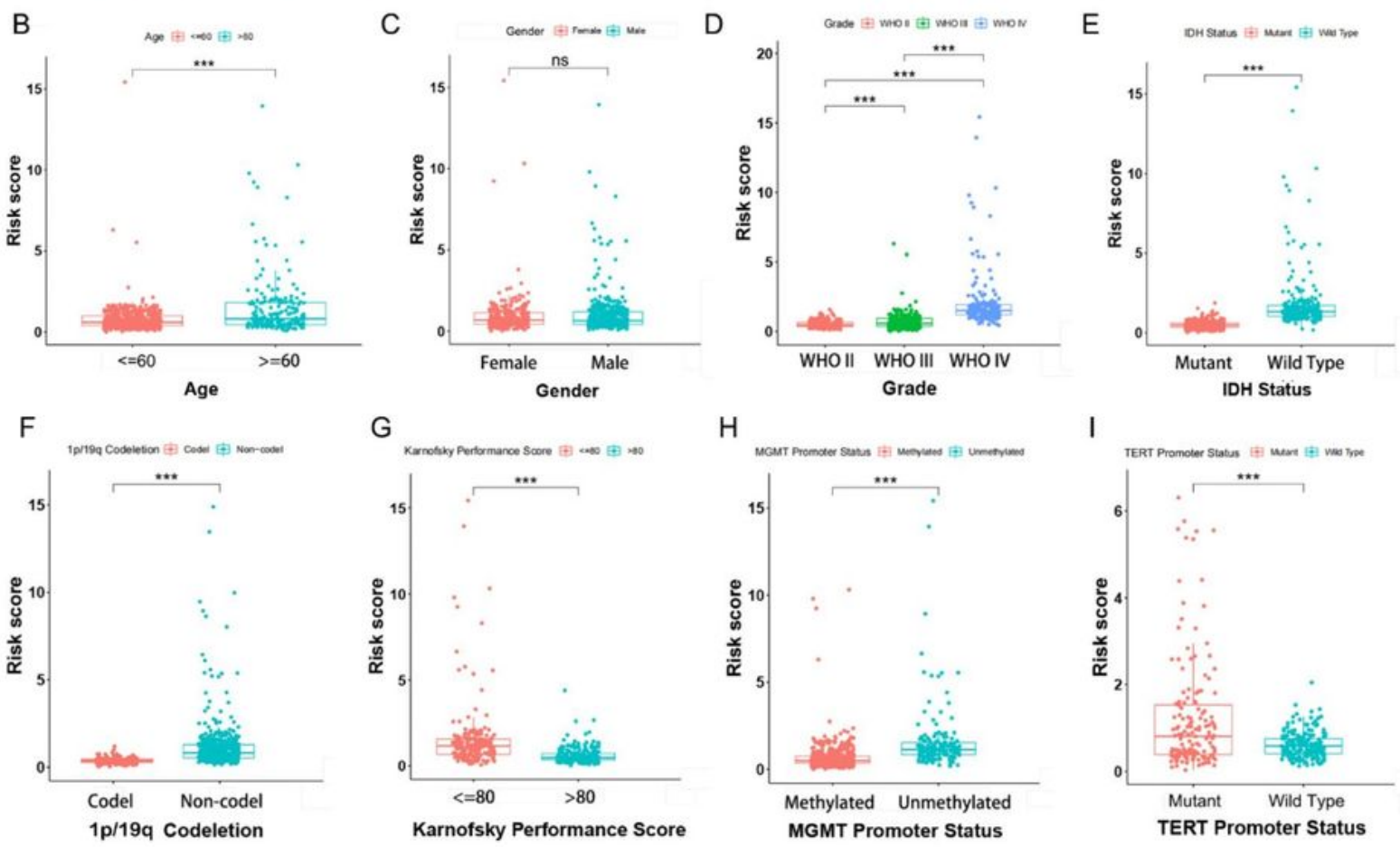

Figure 3

Relationship between risk signature and clinical features of glioma in TCGA database. (A) The heatmap showed the relationship between high and low risk groups and clinical features of glioma patients. Risk 
scores of high and low groups of (B) Age, (C) Gender, (D) WHO grade, (E) IDH mutation status, (F) 1p/19q codeletion, (G) Karnofsky Performance Score, (H) MGMT promoter methylation status and (I) TERT promoter status.

A
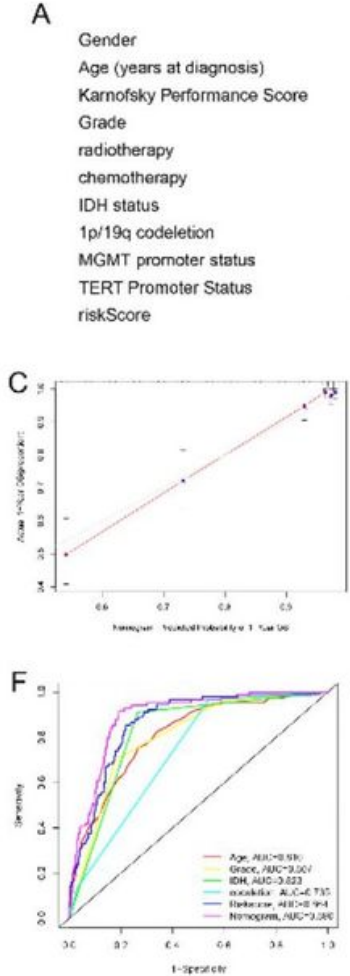
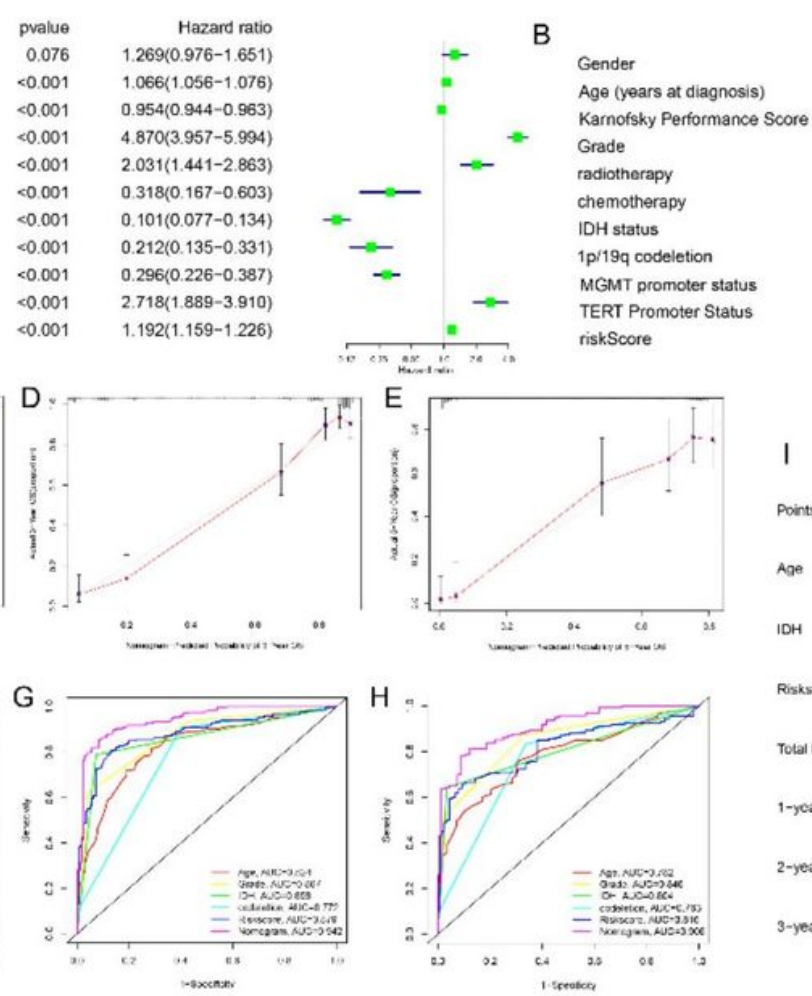

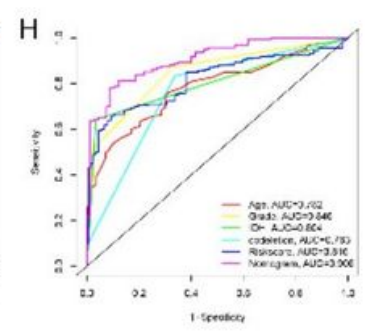

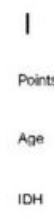

Risksecone

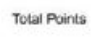

1-year sunvivat

2-year survevo

3-year survival
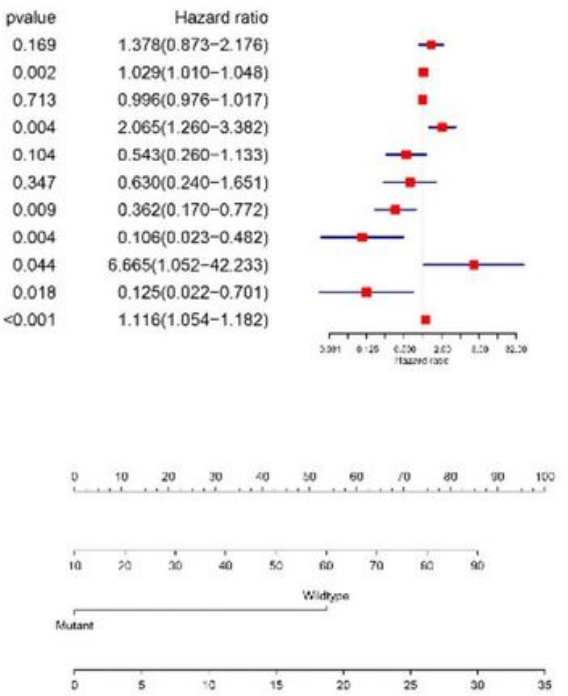

\begin{tabular}{lllllllllllll}
\hline 20 & 40 & 80 & 80 & 100 & 120 & 160 & 180 & 130 & 250 & 220 & 200 & 280
\end{tabular}

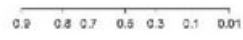

as cos of ob as ar on

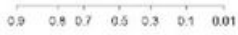

\section{Figure 4}

A nomogram based on risk score and some clinical characteristics was established. (A) Univariate and (B) Multivariate Cox regression analyses were used to verify the prognostic value of risk signature. (C), (D) and (E) Calibration plots for predicting patient 1-year, 3-year and 5-year OS. (F), (G) and (H) ROC curves were used to evaluate the predictive ability of the nomogram and other predictors (1-year, 3-year and 5-year). (I) The nomogram for predicting proportion of patients with 1-year, 3-year and 5-year OS. 

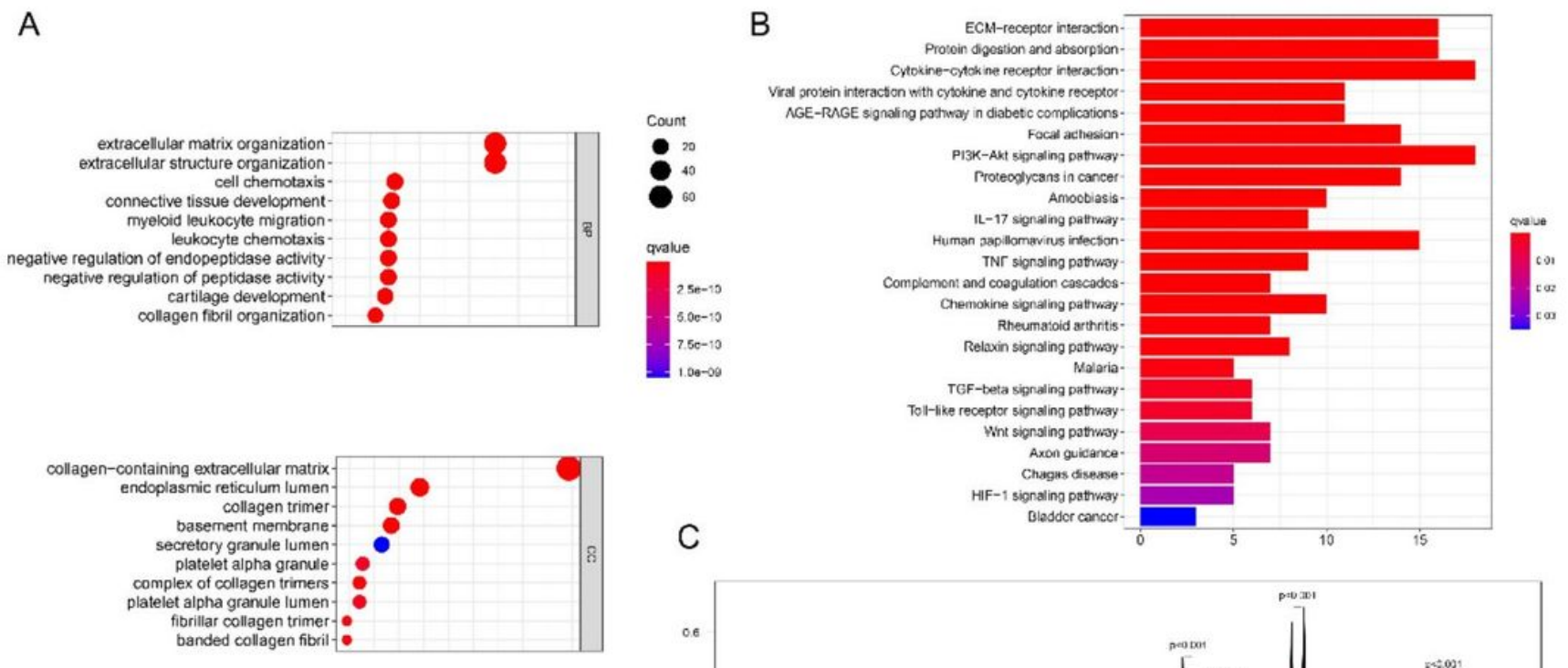

C

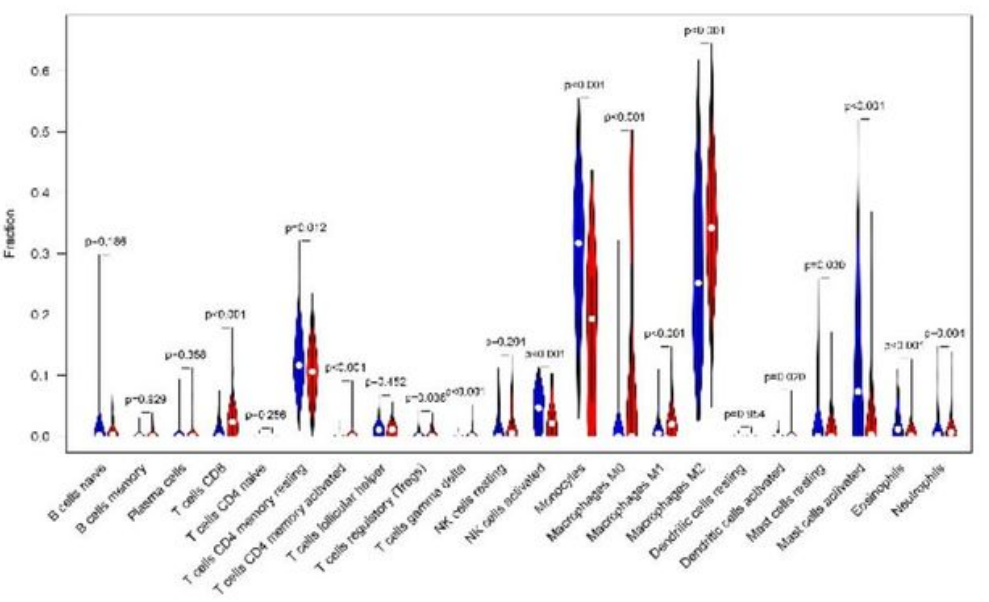

Figure 5

Functional enrichment pathway analysis and immune infiltration of risk signatures. (A) GO analysis. (B) KEGG analysis. (C) Immune cell infiltration in high and low risk groups. 
A

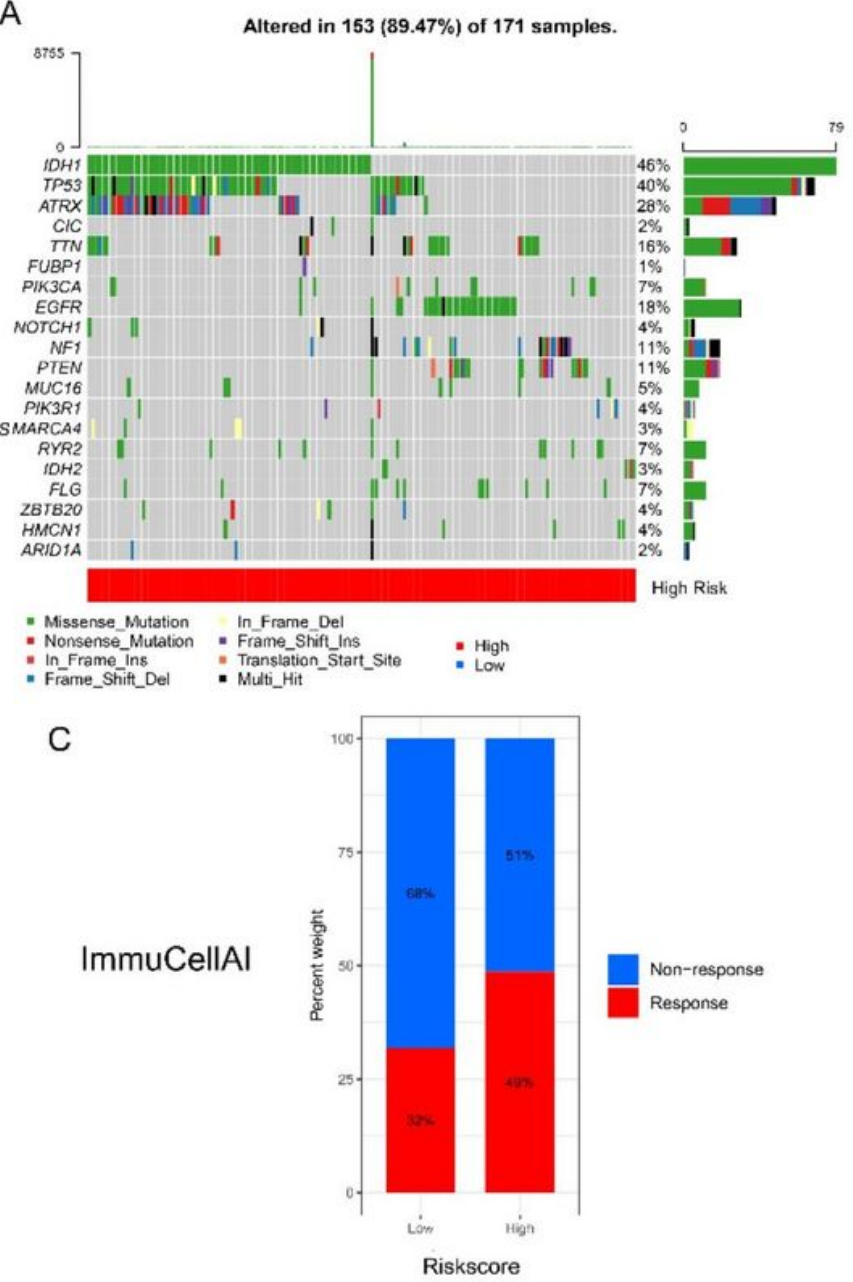

B

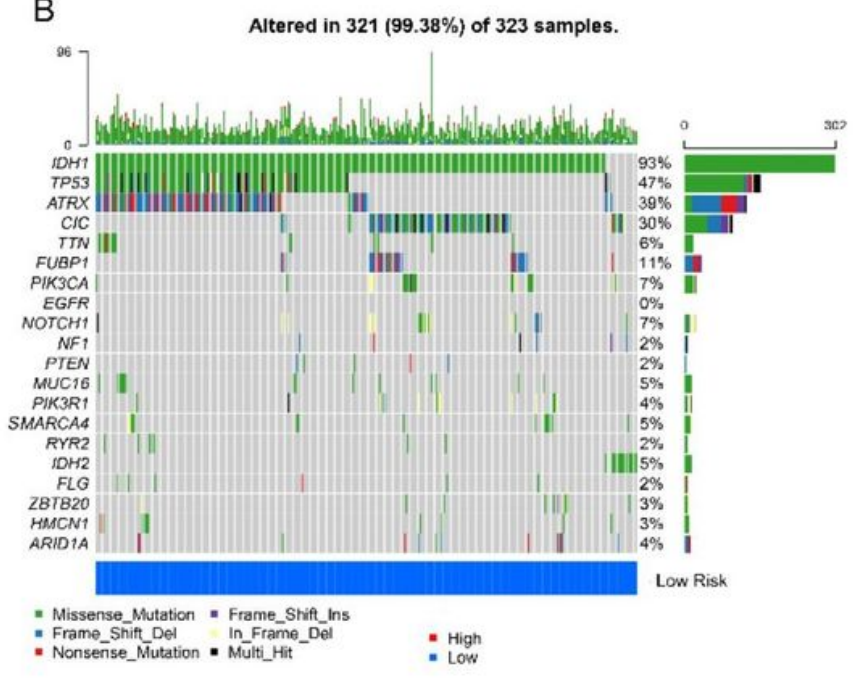

D

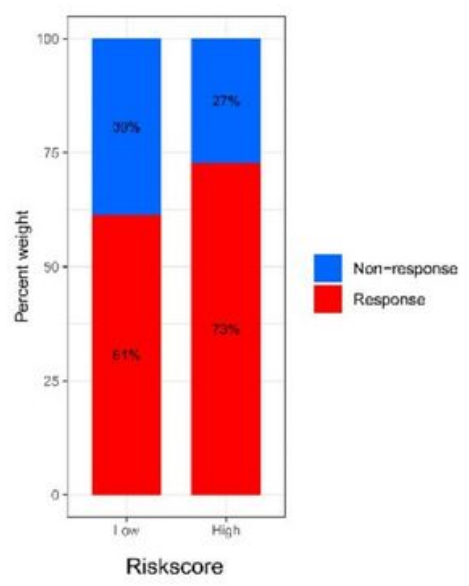

Figure 6

The tumor mutation burdens and anti-PD1/L1 therapy responses of risk signature. (A) Tumor somatic mutation in high-risk group. (B) Tumor somatic mutation in low-risk group. (C) Anti- PD1/L1 immunotherapy response of high and low risk groups in ImmuCellAI. (D) Anti- PD1/L1 immunotherapy response of high and low risk groups in TIDE. 

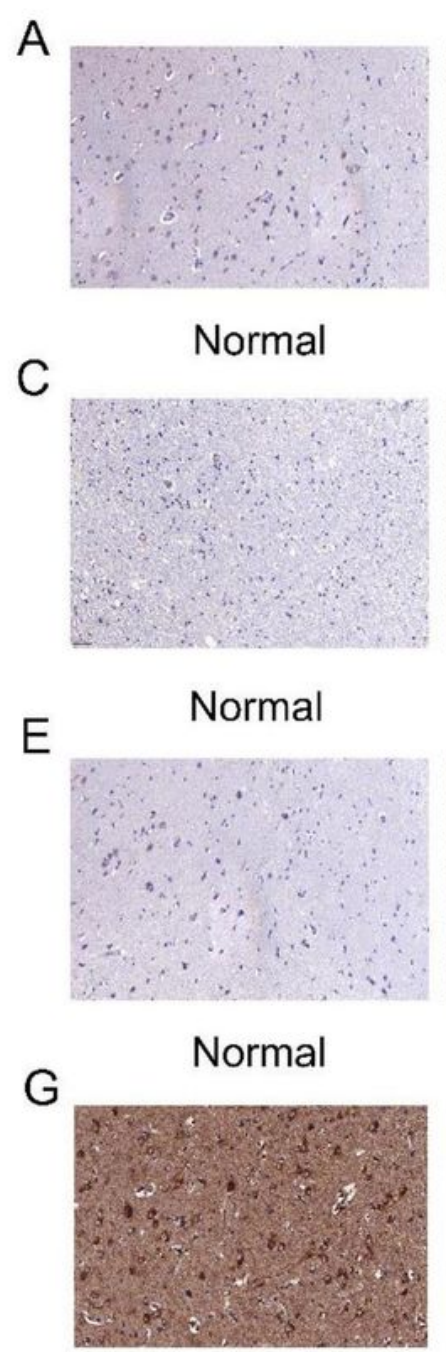

Normal

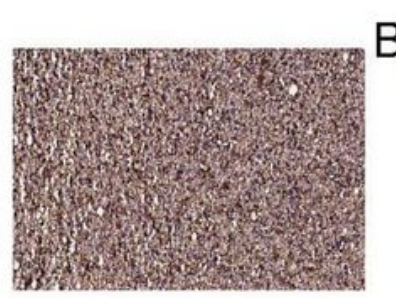

Tumor
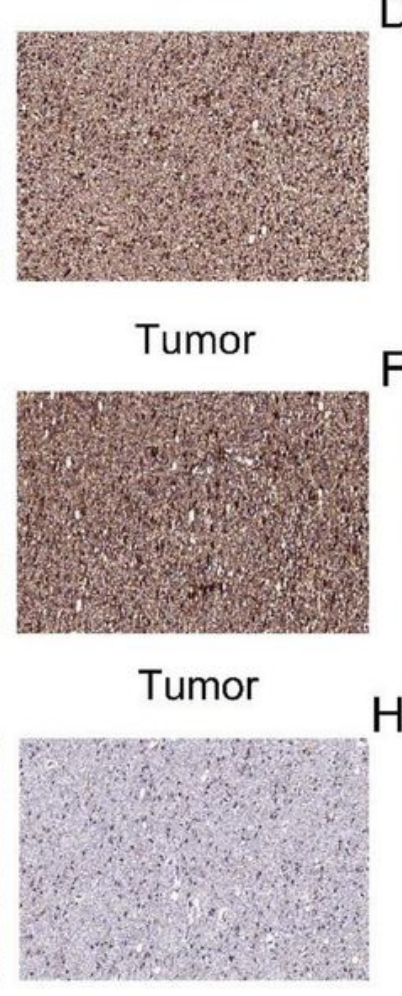

Tumor

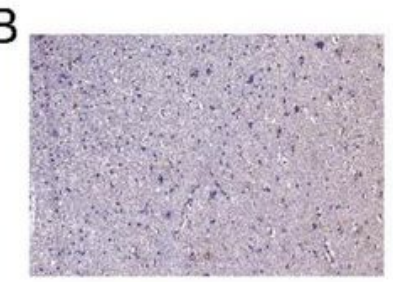

Normal

D

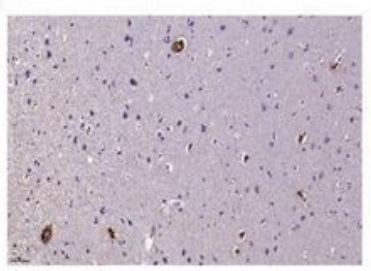

Normal

$F$

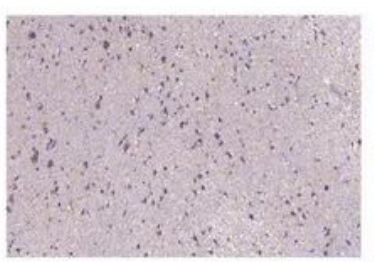

Normal

$\mathrm{H}$

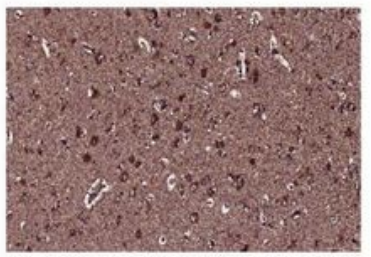

Normal

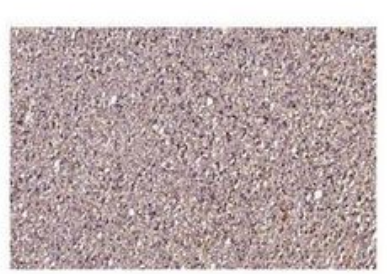

Tumor

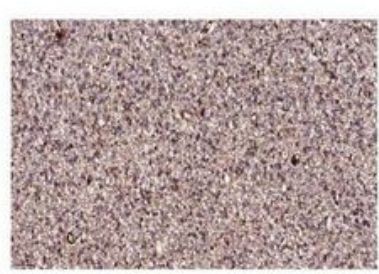

Tumor

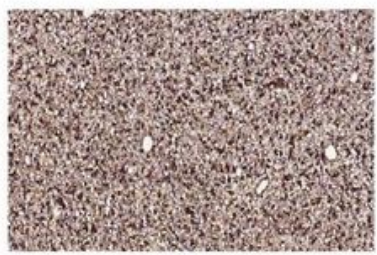

Tumor

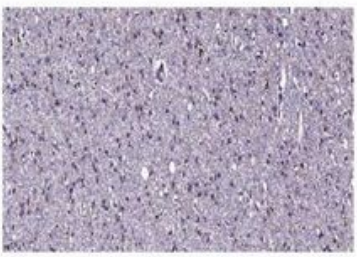

Tumor

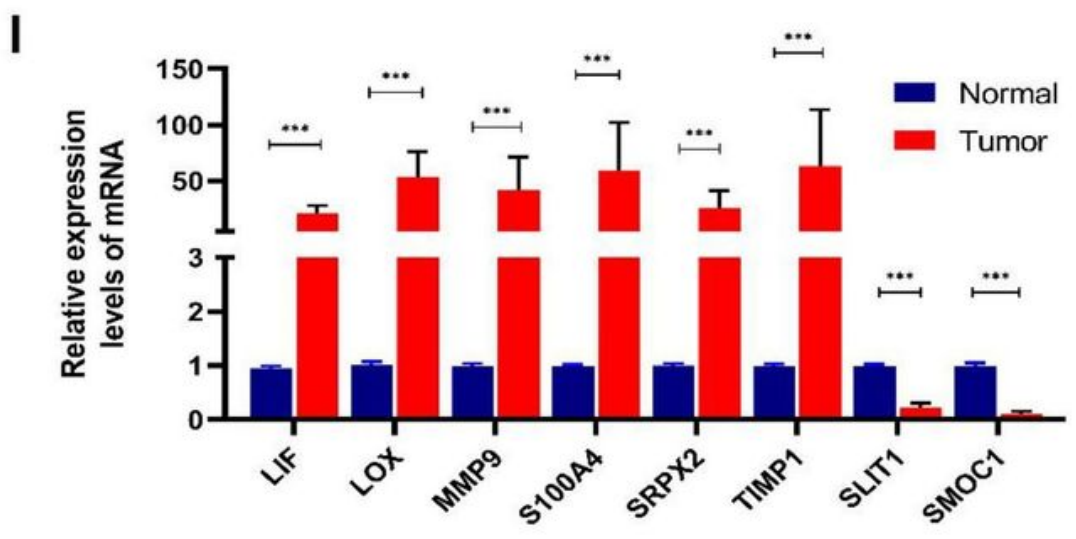

Figure 7

The expression levels of $\mathbf{8}$ immune-related matrisomes in glioma verified by Immunohistochemistry and qPCR. Immunohistochemistry of (A) LIF. (B) LOX. (C) MMP9. (D) S100A4. (E) SRPX2. (F) TIMP1. (G) SLIT1. (H) SMOC1. (I) The mRNA expression levels of 8 immune-related matrisomes (qPCR).

\section{Supplementary Files}


This is a list of supplementary files associated with this preprint. Click to download.

- supplementaryfiles.docx

- SupplementaryFigures.docx 